\title{
V-Series Nerve Agent VP
}

National Cancer Institute

\section{Source}

National Cancer Institute. V-Series Nerve Agent VP. NCI Thesaurus. Code C161537.

An org anophosphate nerve agent, closely related to the nerve agent VX, that is a

persistent, oily liquid at room temperature, is absorbed into the body through inhalation

or skin contact and is an acetylcholinesterase inhibitor and neurotoxin. 